\title{
Glucocorticoids reset circadian clock in choroid plexus via period genes
}

\author{
Karolína Liška1,2, Martin Sládek1, Vendula Čečmanová1 and Alena Sumová(i)1 \\ ${ }^{1}$ Laboratory of Biological Rhythms, Institute of Physiology, Czech Academy of Sciences, Prague, Czech Republic \\ ${ }^{2}$ Third Faculty of Medicine, Charles University, Prague, Czech Republic
}

Correspondence should be addressed to A Sumová: alena.sumova@fgu.cas.cz

\begin{abstract}
The epithelial cells of choroid plexus (CP) in brain ventricles produce cerebrospinal fluid and act as the blood-cerebrospinal fluid barrier. In this study, we confirmed that $\mathrm{CP}$ in the 4th ventricle is composed of cellular oscillators that all harbor glucocorticoid receptors and are mutually synchronized to produce a robust clock gene expression rhythm detectable at the tissue level in vivo and in vitro. Animals lacking glucocorticoids (GCs) due to surgical removal of adrenal glands had Per1, Per2, Nr1d1 and Bmal1 clock gene rhythmicity in their CP significantly dampened, whereas subjecting them to daily bouts of synthetic GC analog, dexamethasone (DEX), reinforced those rhythms. We verified these in vivo effects using an in vitro model of organotypic CP explants; depending on the time of its application, DEX significantly increased the amplitude and efficiently reset the phase of the CP clock. The results are the first description of a PRC for a non-neuronal clock in the brain, demonstrating that CP clock shares some properties with the non-neuronal clocks elsewhere in the body. Finally, we found that DEX exhibited multiple synergic effects on the CP clock, including acute activation of Per1 expression and change of PER2 protein turnover rate. The DEX-induced shifts of the CP clock were partially mediated via PKA-ERK1/2 pathway. The results provide the first evidence that the GC rhythm strengthens and entrains the clock in the $\mathrm{CP}$ helping thus fine-tune the brain environment according to time of day.
\end{abstract}

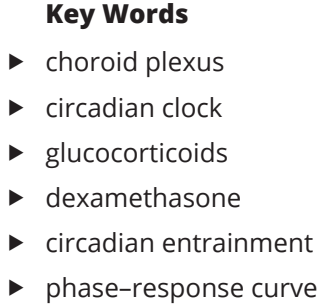

Journal of Endocrinology (2021) 248, 155-166

\section{Introduction}

The choroid plexus (CP), a secretory epithelium located in all four ventricles of the mammalian brain, produces cerebrospinal fluid (CSF) by filtration of blood from fenestrated capillaries. The CP also acts as the so-called blood-cerebrospinal fluid barrier, forms an enzymatic barrier and secretes biologically active substances, thus playing an overlooked role in brain signaling (reviewed in Wolburg \& Paulus 2010). Changes in the production of CSF and its turnover have been implicated in both aging and several neuropsychiatric disorders (reviewed in Skipor \& Thiery 2008). The function of CP and CSF production and turnover were found to be compromised in aged animal models and in patients suffering from neurodegenerative and psychiatric disorders (Silverberg et al. 2003, Wostyn et al. 2011, Chiu et al. 2012). In rats, the CP has also shown changes following chronic stress, a model used to induce depression-like behavior (Sathyanesan et al. 2012).

Importantly, the production and composition of CSF follows a daily rhythm (Nilsson et al. 1992, Harrington et al. 2010). The mechanism of these rhythmic changes is not known but recently, the $\mathrm{CP}$ cells were found to harbor a robust circadian clock (Quintela et al. 2015, 2018, Myung 
et al. 2018, Yamaguchi et al. 2020). At the cellular level, the circadian clocks operate via transcriptional-translational feedback loop mechanism (TTFL) (Takahashi et al. 2008) which rhythmically drives expression of clock genes (e.g. Per1,2, Cry1,2, Bmal1, Nr1d1) and temporally controls expression of tissue-specific physiologically relevant genes. The clock in the $\mathrm{CP}$ is self-autonomous because it can run in absence of any rhythmic input, as demonstrated in organotypic explants from $m P e r 2^{\text {luc }}$ mice which maintain bioluminescence rhythms for several days in culture (Myung et al. 2018, Quintela et al. 2018, Yamaguchi et al. 2020). Nevertheless, similar to clocks in other brain regions and elsewhere in the body (Dibner et al. 2010), the CP clock is dependent on signals controlled by the central clock in the suprachiasmatic nuclei (SCN) for proper alignment with environmental cycles. These signals have not been identified. Theoretically, they may involve SCN-controlled neuronal pathways because $\mathrm{CP}$ receives autonomic innervation, with the sympathetic nervous system reducing the CSF secretion and the cholinergic system increasing it (Lindvall \& Owman 1981), as well as rhythmically varying levels of hormones and other signaling molecules carried by blood and CSF. The aim of our study was to test the hypothesis that glucocorticoids (GCs) play a role in the entrainment of the CP clock because their production in adrenal glands is controlled by the SCN in alignment with the sleep/wake state (Cheifetz 1971). The GCs are strong candidates also because the CP cells contain glucocorticoid receptors (GRs) (Sinclair et al. 2007, Martinho et al. 2012), and GCs synchronize circadian clocks in various cellular models, tissues and organs (reviewed in Spencer et al. 2018) mostly via a direct effect on transcription of clock genes which contain glucocorticoid responsive elements (GREs) in their promoters (Torra et al. 2000, So et al. 2009). To test our hypothesis, we used approaches that addressed (1) the impact of the absence of endogenous GCs on the CP clock in vivo, (2) the ability of GCs to entrain the CP clock in vitro, and (3) the effect of GCs on transcriptional and post-translational processes in the CP clock. Our results are strongly in favor of the proposed hypothesis and suggest the involvement of both the conventional transcriptional regulation via GRE as well as novel signaling pathways connecting the GC signaling with the CP clock.

\section{Materials and methods}

\section{Animals}

Adult male Wistar rats (Institute of Physiology, the Czech Academy of Sciences) and adult $m P e r 2^{L u c}$ mice of both genders (strain B6.129S6-Per2tm1Jt/J, JAX, USA; a colony maintained at the Institute of Physiology, the Czech Academy of Sciences) were housed individually under a $12 \mathrm{~h}$ light:12 h darkness cycle (LD12:12); light on at 06:00 h corresponded to Zeitgeber time (ZT) 0 , light off at 18:00 h corresponded to ZT12. Food and water provided ad libitum. All experiments were approved by the Animal Care and Use Committee of the Institute of Physiology and were in agreement with the Animal Protection Law of the Czech Republic, as well as the European Community Council directives 86/609/EEC. All efforts were made to lessen the suffering of animals.

\section{In vivo experiments}

The rats were subjected to adrenalectomy or sham surgery (the same procedure but without removal of adrenal glands) according to the method described previously (Soták et al. 2016). The adrenalectomized animals had free access to $0.9 \% \mathrm{NaCl}$ drinking solution. They were either untreated (ADX; $n=35$ ) or treated with dexamethasone (intraperitoneal injections; $1 \mathrm{mg} / \mathrm{kg}$ b.w.) at ZT12 (18:00 h) for 8 days (ADX+DEX; $n=27)$. The sham-operated rats (SHAM; $n=35$ ) were untreated and used as controls. Eight days after surgery, the lights were not switched on and the rats were sacrificed under deep isoflurane anesthesia in constant darkness in $4 \mathrm{~h}$ intervals during $24 \mathrm{~h}$ (4-5 animals per time point). The brains were frozen on dry ice and kept at $-80^{\circ} \mathrm{C}$ until detection of mRNA levels using in situ hybridization. Time was expressed as circadian time (CT) with time zero corresponding to the previous lights-on. The complete abolishment of endogenous GCs in ADX animals was shown elsewhere (Soták et al. 2016).

For detection of acute responses to DEX, intact rats maintained in $12 \mathrm{~h}$ light: $12 \mathrm{~h}$ darkness cycle were treated with DEX ( $1 \mathrm{mg} / \mathrm{kg}$ b.w., i.p.; $n=30)$ or vehicle (VEH; PBS; $n=30) 4 \mathrm{~h}$ after lights off (ZT16), that is, when DEX application resulted in significant changes in the CP clock phase (for more details, see 'Results' section). Animals were sacrificed by rapid decapitation under deep anesthesia (i.p. injections of sodium thiopental $50 \mathrm{mg} / \mathrm{kg}$ b.w.) at 0 , 1,2 and $4 \mathrm{~h}$ following the VEH or DEX application (five animals per time point and group). The brains were frozen on dry ice and kept at $-80^{\circ} \mathrm{C}$ until isolation of the 4 th ventricle CPs for RT-qPCR assay.

\section{In situ hybridization}

Frozen rat brain coronal sections containing the 4th ventricle $\mathrm{CP}$ were processed for in situ hybridization as 
previously described (Sumová et al. 2003) to determine the expression of Per1 (980 bp, AB002108), Per2 (1512 bp, NM_031678), Nr1d1 (1109 bp, BC062047) and Bmal1 (841 bp, AB012600) in the CP.

\section{RT-qPCR in laser-dissected CP regions}

Frozen rat brains were sectioned on cryostat into $30-\mu \mathrm{m}$ coronal sections. CP was separated from the 4 th ventricle using a laser microdissector (LMD6000, Leica), total RNA was isolated and reverse-transcribed into cDNA as described previously (Houdek \& Sumová 2014). The cDNA samples were analyzed by RT-qPCR using 5x HOT FIREPol Probe qPCR mix Plus (Solis Biodyne; Tartu, Estonia) and TaqMan Gene Expression Assays (Life Technologies) spanning exon junctions specific for rat genes Gilz (Rn00580222 m1), Per1 (Rn01325256_m1), Per2 (Rn01427704_m1), Nr1d1 (Rn01460662_m1) and Bmal1 (Rn00577590_m1). The mRNA concentrations were normalized relative to the Beta-2-Microglobulin (Rn00560865_m1) housekeeping gene expression, measured in a duplex reaction. Relative cDNA concentrations were quantified using the Pfaffl $\Delta \Delta$ Ct method.

\section{Immunohistochemistry}

Frozen rat brain sections were processed for immunohistochemistry with rabbit GR polyclonal primary IgG antibody with epitope mapping at the N-terminus of GR $\alpha$ (M-20, CAT\#:sc-1004, RRID:AB_2155786, Santa Cruz Biotechnology) as described elsewhere (Sumová et al. 2002). Final antibody concentration used was 1:100. Pictures were taken using Leica SP8 WLL MP laser scanning confocal microscope.

\section{Organotypic explants and bioluminescence recordings}

mPer2 ${ }^{\text {Luc }}$ mice were sacrificed between 12:00 and 15:00 h and brains were sectioned into 300- $\mu \mathrm{m}$ coronal sections using vibratome (Leica). CP explants were dissected from the 4th ventricle and immediately placed onto Millicell Culture Inserts (Merck) inside 35-mm petri dishes containing $1 \mathrm{~mL}$ of air-buffered recording media (DMEM supplemented with $100 \mathrm{U} / \mathrm{mL}$ penicillin, $100 \mu \mathrm{g} /$ mL streptomycin, 1\% GlutaMAX (all Thermo Fisher), 2\% B27 supplement (Thermo Fisher) and $0.1 \mathrm{mM}$ D-luciferin (Biosynth, Staad, Switzerland). The bioluminescence traces were recorded in Lumicycle (Actimetrics, Wilmette, IL, USA) and analyzed using LumiCycle Analysis software
(Actimetrics). The data were baseline-subtracted using the 24-h running average and fitted with a damped sine wave to calculate the period, amplitude and phase.

\section{In vitro response to dexamethasone application}

The organotypic explants of the 4 th ventricle CP were cultured in fresh recording media. During the 4th day they were exposed to a treatment procedure (either $1 \mu \mathrm{L}$ of $100 \mu \mathrm{M}$ DEX per $1 \mathrm{~mL}$ of media or $1 \mu \mathrm{L}$ of the corresponding vehicle: $0.001 \%$ ethanol in ddH2O) at different times relative to the phase of bioluminescence rhythm. A separate set of explants treated with DEX were pretreated with $1 \mu \mathrm{L}$ of selected inhibitors (mifepristone (MIF): $1 \mathrm{mM}, n=24$, Chelerythrine (Chel): $10 \mathrm{mM}, n=17$, H89: $10 \mathrm{mM}, n=13$, U0126: $25 \mathrm{mM}, n=17$ ) or VEH (ethanol for MIF, $n=23$, water for Chel, $n=54$ and H89, $n=32$, DMSO for U0126, $n=15$ ). For repeated treatments, the explants were washed with $37^{\circ} \mathrm{C}$ PBS (two times, 5 $\mathrm{min}$ ), synchronized using serum shock ( $2 \mathrm{~h}$ in $50 \%$ horse serum, $37^{\circ} \mathrm{C}$ ), washed again and placed in fresh media. Relative amplitudes and periods were calculated as ratios of the amplitude and period values detected after the treatment and prior to the treatment. The phase shifts were quantified by fitting a sine curve to at least three full circadian cycles of a 24 -h running average baselinesubtracted rhythm and then extrapolating beyond the time of treatment. The calculated phase shift was designated as a phase advance $(+)$ or a delay $(-)$. The phase-response curves (PRCs) were constructed by plotting the calculated phase shift as a function of treatment time normalized to the endogenous period in vitro and expressed relative to the trough (time 0 ) or peak (time 12) of the rhythm. The effects of inhibitor or VEH pretreatment before DEX on the phase were plotted as PRCs.

\section{PER2LUC degradation assay}

The 4th ventricle CP explants were synchronized using serum shock (see previously), cultured in fresh recording media in Lumicycle until the first peak in PER2-driven bioluminescence was reached. Cycloheximide (Sigma Aldrich) (CHX, $40 \mu \mathrm{g} / \mathrm{mL})$, an inhibitor of translation, was added either alone (CHX; $n=10)$ or in combination with $1 \mu \mathrm{L}$ of $100 \mu \mathrm{M} \operatorname{DEX}(\mathrm{CHX}+\mathrm{DEX} ; n=10)$ and the bioluminescence was recorded for at least $8 \mathrm{~h}$. Onephase exponential decay curves were fitted. Afterwards, the explants were washed, placed into fresh media and immediately recorded in Lumicycle. The rate of PER2 accumulation was expressed as the time between the wash 
A
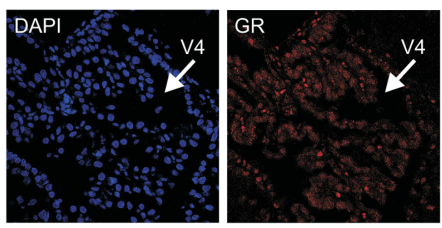

C ADX
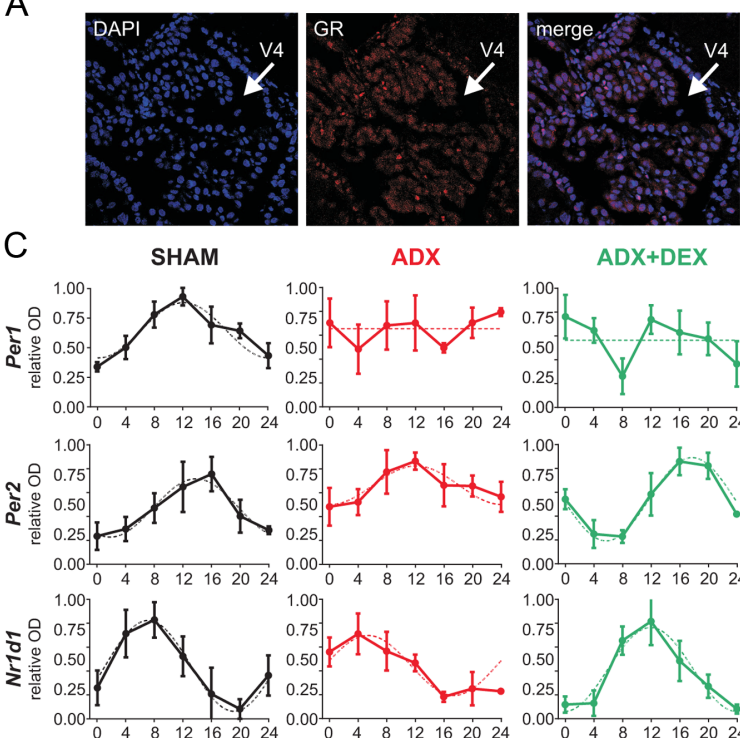

$A D X+D E X$
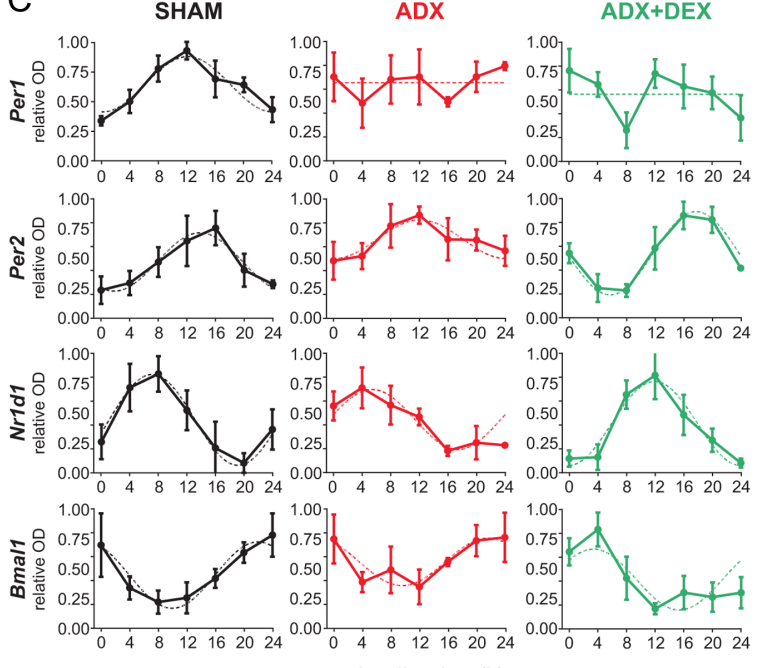

circadian time $(\mathrm{h})$
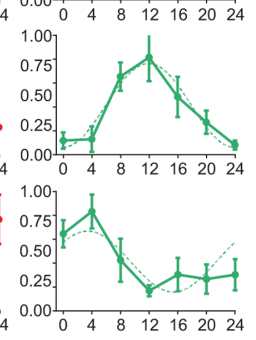
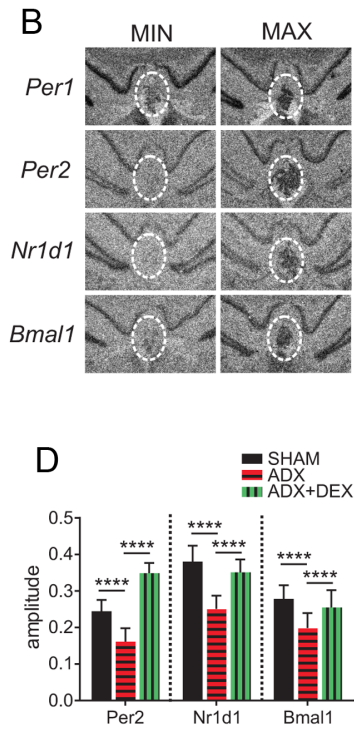

Figure 1

Endogenous GCs are necessary for the robustness of $\mathrm{CP}$ clock in vivo. (A) Representative coronal brain sections demonstrating GR immunopositivity in cells of the rat CP. (B) Representative autoradiographs showing trough and peak of Per1, Per2, Nr1d1 and Bmal1 expression in the $C P$ (indicated by dashed white area) of $A D X+D E X$ group. (C) Daily profiles of relative Per1, Per2, Nr1d1 and Bmal1 mRNA in CP of SHAM (left), ADX (middle) and ADX+DEX (right) groups detected by in situ hybridization ( $n=4-5$ animals per time point). The data were normalized to the maximal value of each data set (plotted as 1), and fitted with cosine curves. Data are shown as mean \pm S.D. (D) Amplitudes of Per2, Nr1d1 and Bmal1 gene expression calculated using the cosinor analysis were compared between the SHAM, ADX and ADX+DEX groups by one-way ANOVA with Sidak's post hoc test: $* \star \star \star P P$ $<0.0001$. Data are shown as mean \pm s.D. A full color version of this figure is available at https:// doi.org/10.1530/JOE-20-0526. and the first peak in the bioluminescence of individual explants.

\section{Statistical analysis}

Daily profiles of normalized clock gene expression were analyzed for rhythmic expression by fitting two alternative regression models: horizontal straight line (null hypothesis) or cosine curve defined by the equation $\mathrm{Y}=$ mesor + (amplitude $\times \cos (2 \times \pi \times(\mathrm{X}-$ acrophase $) /$ wavelength)) with a constant wavelength of $24 \mathrm{~h}$ (alternative hypothesis). $P$ values, $\mathrm{R}^{2}$ (goodness of fit), amplitudes and acrophases were determined. Amplitudes calculated by cosinor analysis were compared between groups by one-way ANOVA with Sidak's post hoc test. Fold changes in gene expression detected after acute DEX injection in vivo based on VEH controls were analyzed using two-way ANOVA. The relative periods and amplitudes of organotypic explants were compared by unpaired t-test; the periods before and after treatment were evaluated by paired t-test. The PRCs were binned into $3 \mathrm{~h}$ intervals, and compared using two-way ANOVA (effect of group) with the post hoc analysis of Sidak's multiple comparison method. The effect of inhibitor vs VEH pretreatment to DEX application was tested using F-test comparing linear regression curves fitted to the phase responses. For $P<0.05$ a single curve, and for $P>0.05$ two separate curves were plotted. The half-life and rate constant (K) of one-phase exponential decay curves of PER2 degradation in organotypic explants and the accumulation times were compared using an unpaired t-test.

All statistics were performed using GraphPad Prism 7 software (GraphPad).

\section{Results}

\section{Endogenous GCs are required for robustness of the circadian clock in CP in vivo}

We first confirmed the presence of GRs in the cytoplasm of all cells of rat $\mathrm{CP}$ by immunohistochemistry (Fig. 1A). Then, we tested the impact of endogenous GCs absence on the clocks in the rat CP. The animals were either sham-operated (SHAM) or exposed to surgical ablation of adrenal glands (ADX). Daily expression profiles of Per1, Per2, Nr1d1 and Bmal1 in the CP were assessed using in situ hybridization (representative autoradiographs are shown in Fig. 1B). Daily clock gene expression profiles (Fig. 1C) in the SHAM group exhibited robust circadian rhythms with mutual phases as expected according to the TTFL model, that is, Bmal1 and Nr1d1 acrophases in antiphase, and the Per1 acrophase delayed to Nr1d1 and advanced to Per2 (Table 1 - cosinor rhythms acrophases). The ADX completely abolished the rhythm in Per1 expression and significantly reduced 
Table 1 Cosinor analysis of the rat CP clock gene expression profiles.

\begin{tabular}{l}
\hline \\
\hline SHAM \\
acro \pm SE \\
amp \pm SE \\
$\mathrm{R}^{2}$ \\
$P$ \\
ADX \\
acro \pm SE \\
amp \pm SE \\
$\mathrm{R}^{2}$ \\
$P$ \\
ADX+DEX \\
acro \pm SE \\
amp \pm SE \\
$\mathrm{R}^{2}$ \\
$P$
\end{tabular}

\begin{tabular}{c}
\hline Per1 \\
\hline $12.15 \pm 0.54$ \\
$0.234 \pm 0.032$ \\
0.712 \\
$<0.0001$ \\
- \\
- \\
0.032 \\
0.619 \\
- \\
- \\
0.08 \\
0.3123 \\
\hline
\end{tabular}

\begin{tabular}{c}
\hline Per2 \\
\hline $13.81 \pm 0.52$ \\
$0.244 \pm 0.031$ \\
0.660 \\
$<0.0001$ \\
$12.08 \pm 0.95$ \\
$0.162 \pm 0.037$ \\
0.439 \\
0.0007 \\
$17.69 \pm 0.30$ \\
$0.349 \pm 0.028$ \\
0.850 \\
$<0.0001$
\end{tabular}

\begin{tabular}{c}
\hline Nr1d1 \\
\hline $8.84 \pm 0.39$ \\
$0.380 \pm 0.043$ \\
0.727 \\
$<0.0001$ \\
$5.37 \pm 0.59$ \\
$0.251 \pm 0.036$ \\
0.665 \\
$<0.0001$ \\
$11.91 \pm 0.39$ \\
$0.351 \pm 0.035$ \\
0.799 \\
$<0.0001$ \\
\hline
\end{tabular}

\begin{tabular}{c}
\hline Bmal1 \\
\hline $22.08 \pm 0.54$ \\
$0.278 \pm 0.038$ \\
0.642 \\
$<0.0001$
\end{tabular}

Acrophases (acro) in hours and amplitudes (amp) are shown for daily profiles with significant cosinor fits as assessed by $\mathrm{R}^{2}$ and significance level $(P)$ in control (SHAM), adrenalectomized (ADX) and adrenalectomized and injected with dexamethasone (ADX+DEX) animals. Data are mean \pm S.E.M.

robustness of the circadian rhythms in expression of Per2, Nr1d1 and Bmal1 (Table 1 - cosinor rhythms amplitudes; for statistics, see also Fig. 1D - SHAM vs ADX). Daily injections of ADX animals with DEX (1 mg per kg, i.p. before lightsoff) for 8 days preceding collection of samples (ADX+DEX) strengthened circadian rhythmicity via increasing the amplitudes of rhythms in expression of Per2, Nr1d1, and Bmal1 (Table 1 - cosinor rhythms amplitudes; for statistics, see Fig. 1D - ADX vs ADX+DEX). Although cosinor analysis did not confirm rhythm in Per1 expression in ADX+DEX group (Table 1), there was an apparent significant elevation in Per1 mRNA levels at the time corresponding to the DEX treatment (between circadian time 8 and 12) (one-way ANOVA; $P=0.0004$, multiple comparisons; $P=0.0001$ ) suggesting an acute response of the gene to the DEX administration. The phases of the DEX-reinforced Per2, Nr1d1 and Bmal1 expression rhythms (Table 1 - cosinor rhythms acrophases) were shifted compared to those in controls and, importantly, they corresponded to the TTFL model (Nr1d1 preceding Per 2 and being in antiphase to Bmal1). Therefore, the ADX suppressed, and the daily DEX injections reinforced, the $\mathrm{CP}$ clock in vivo.

\section{DEX resets the CP clock in vitro}

Next, we tested the effect of DEX directly on parameters of the CP clock in real-time in vitro using organotypic 4 th ventricle CP explants of $m P e r 2^{L u c}$ mice that exhibited circadian rhythm in PER2-driven bioluminescence (Fig. 2) with the period of $26.39 \pm 0.05 \mathrm{~h}$ (mean \pm s.E.M.; $n=268$ ). On the 5th day in culture, the explants were treated with vehicle (VEH; $0.001 \%$ ethanol in ddH2O; $n=103$ ) or DEX
(100 nM; $n=248$ ) and the bioluminescence was recorded for 4 more days (Fig. 2A). The effect of these treatments on amplitudes and periods of the rhythms was assessed using ratios of these parameters before and after each treatment (relative amplitude/period changes in Fig. 2B and C). Compared to VEH, the treatment with DEX significantly increased amplitudes of the rhythms (relative amplitudes: VEH $0.6438 \pm 0.0156$ vs DEX $1.230 \pm 0.0368 ; P<0.0001$ ) (Fig. 2A and B). None of these treatments systematically changed periods of the rhythms, and the relative period changes produced by VEH $(0.9879 \pm 0.0038)$ and DEX $(0.9863 \pm 0.0024)$ treatments did not statistically differ $(P=0.7065)$ (Fig. 2C). Most importantly, the VEH and DEX treatments significantly differed in their effects on phases of these rhythms, whose magnitudes and directions were highly dependent on timing of these treatments. In order to compare between the effects of both treatments, we constructed full phase-response curves (PRCs) (Fig. 2D) by application of VEH or DEX at various time points and plotting the magnitudes and directions of resulting phase shifts as a function of the treatment time expressed relatively to the peak of PER2-driven bioluminescence rhythm (assigned as time 12). VEH treatments sporadically induced only relatively very small phase shifts of the PER2-driven bioluminescence rhythm. In contrast, the DEX treatments produced a typical type 0 PRC (Johnson 1992) with no dead zone and the largest phase shifts up to $12 \mathrm{~h}$ around the transition point matching the peak of the PER2-driven bioluminescence (time 12). Statistical comparison of the PRCs binned into $3 \mathrm{~h}$ intervals confirmed significant differences between the effects of VEH and DEX (two-way ANOVA; $P<0.0001$ ) (Fig. 2E). 

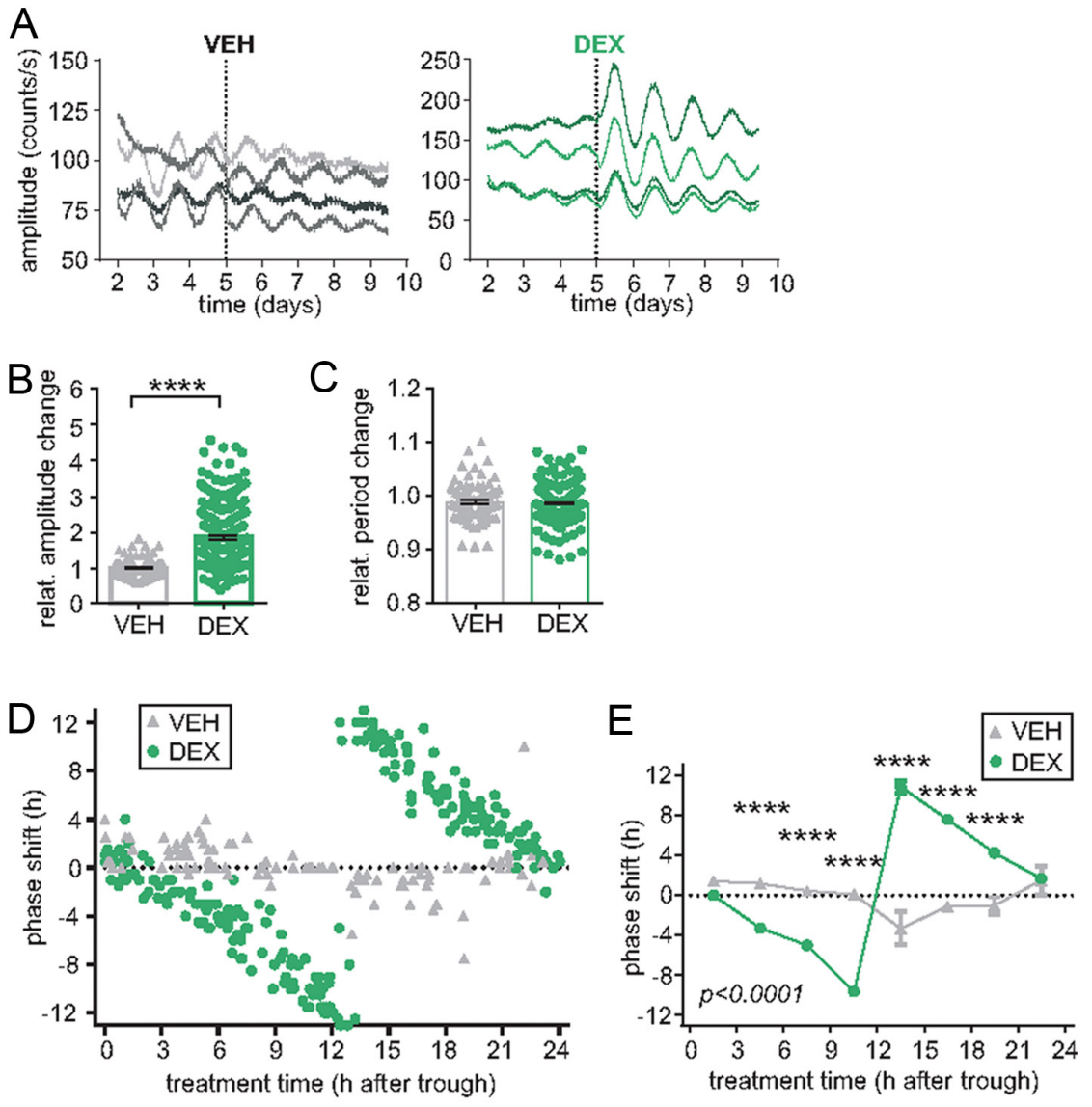

Figure 2
DEX resets the CP clock in vitro. (A) Representative
traces of bioluminescence of CP organotypic
explants from mPer2Luc mice treated with VEH or
DEX (treatment is depicted by the dotted line).
Each trace corresponds to one explant. (B)
Changes in amplitudes of the bioluminescence
rhythms of the CP explants after VEH ( $n=103$ ) or
DEX ( $n=238$ ) treatments. Relative amplitude
changes expressed as individual ratios and mean
\pm s.E.M. Unpaired t-test; $* \star \star * P<0.0001$. (C)
Relative period changes of the bioluminescence
rhythms of the CP explants following VEH ( $n=86$ )
or DEX ( $n=201$ ) treatments. Relative period
changes expressed as individual ratios and mean
\pm s.E.M. Unpaired t-test; $P=0.7065$. (D) Phase-
response curves (PRCs) for VEH ( $n=103$ ) and DEX
( $n=248$ ) treatments of the CP explants. (E)
Comparison of the PRCs for VEH/DEX binned into
3-h intervals is depicted (for all binned time
points, $n \geq 5$ ). Data are mean \pm s.E.M. Two-way
ANOVA ( $P<0.0001$ ) with Sidak's post hoc
comparison: $* \star \star \star P<0.0001$ (F) Effect of
glucocorticoid receptor antagonist mifepristone
(MIF) on the DEX-induced phase shifts. The
DEX-treated CP explants were pretreated with MIF
$(n=24$ ) or VEH ( $n=23$ ). Responses to DEX alone
were replotted ( $n=238$; data in D). (G) Effect of
MIF treatment alone (circles, $n=13$ ) was
compared to the effects of MIF+DEX (squares,
$n=24$ ) on phase of the PER2-driven
bioluminescence in the CP explants. The resulting
phase shifts were not significantly different
between both groups when compared by
unpaired t-test. A full color version of this figure is
available at https://doi.org/10.1530/JOE-20-0526.

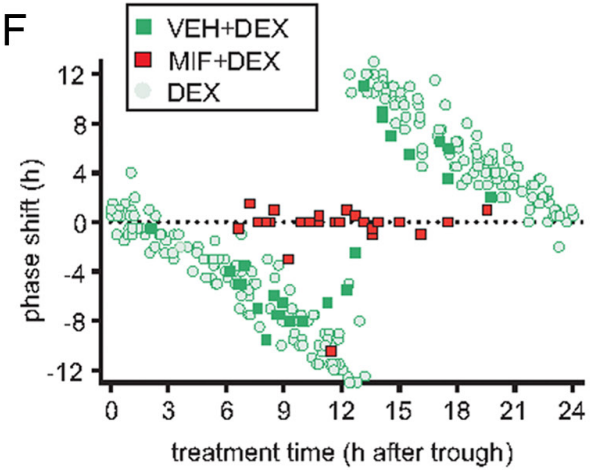

G

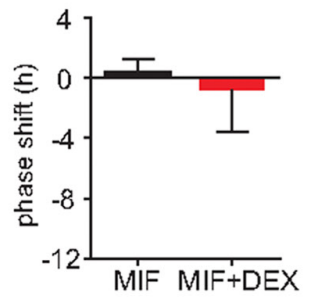

Finally, we addressed the specificity of the treatment effect on the CP clock via testing whether a blockade of GRs will prevent the DEX-induced phase shifts. We applied GR antagonist mifepristone (MIF; $1 \mu \mathrm{M}$ ) before each of the DEX treatments to construct the PRC (Fig. 2F). MIF treatment antagonist completely blocked the effect of DEX on the CP clock so that no shifts were induced throughout the circadian cycle. Treatment of explants with MIF alone had no effect on the phase of the CP clock (Fig. 2G). The data clearly confirmed that the CP clock can be reset by DEX in vitro via GR-dependent pathway.

\section{DEX acutely induces expression of Per1 mRNA in CP}

To explore the mechanisms underlying the DEX-mediated resetting of the $\mathrm{CP}$ clock, we assessed responses in the expression of GC-responsive gene (Gilz) and selected clock genes (Per1, Per2, Nr1d1, Bmal1, E4bp4) to DEX injections to intact rats in laser dissected samples of the CPs using RT-qPCR (Fig. 3A). The rats were sacrificed before (time $0)$, and then 1, 2 and $4 \mathrm{~h}$ after a single injection of DEX (1 $\mathrm{mg}$ per $\mathrm{kg}$, i.p.) or $\mathrm{VEH}$ (corresponding volume of PBS). Compared to the VEH treatment, DEX induced 
A
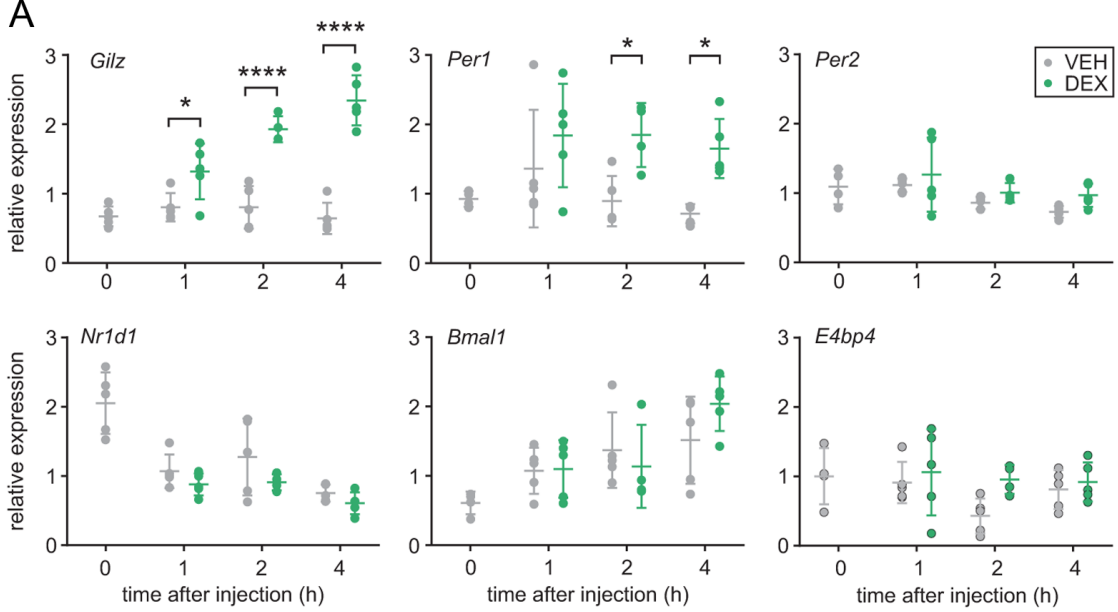

$\mathrm{B}$
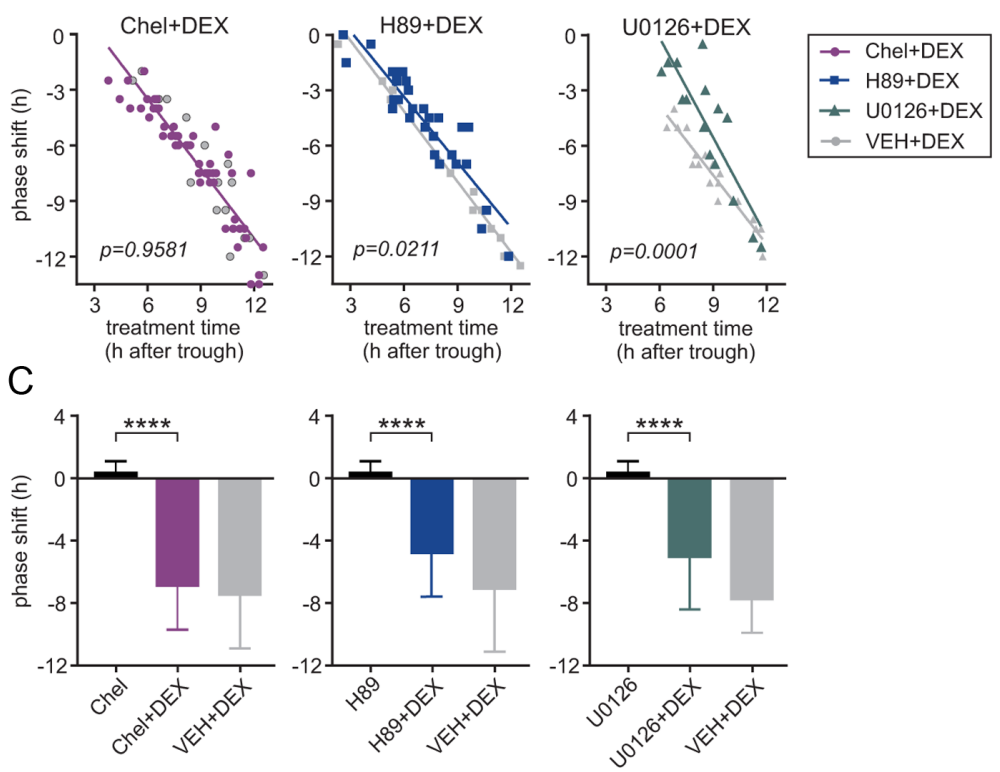

Figure 3

Mechanisms involved in the DEX-induced resetting of the CP clock. (A) Effect of acute DEX injection on gene expression in rat CP was detected in laser dissected CP samples by RT-qPCR. Relative expression of Gilz, Per1, Per2, Nr1d1, Bmal1 and E4bp4 mRNA at 0, 1, 2 and $4 \mathrm{~h}$ after DEX/VEH injection was compared with the baseline values from animals injected with VEH $(n=5)$. Data are shown as mean \pm S.D. (B) Effect of selected inhibitors on the DEX-induced phase changes of the bioluminescence rhythms of the $\mathrm{CP}$ explants from $\mathrm{mPer} \mathrm{Luc}^{\mathrm{Lu}}$ mice. The phase responses to pretreatment with chelerythrine (Chel+DEX; $n=54), \mathrm{H} 89$ (H89+DEX; $n=32$ ) and U0126 (U0126+DEX; $n=15$ ) were fitted with linear regression and compared to VEH pretreatment $\left(\mathrm{VEH}+\mathrm{DEX} ; n_{\text {Chel }}=17, n_{\mathrm{H} 89}=13, n_{\text {U0126 }}=17\right.$, $\left.n_{\text {CHIR }}=18, n_{\text {LiCl }}=16\right)$ using $F$ test. $P_{\text {Chel }}=0.9581$; $P_{\mathrm{H} 89}=0.0211 ; P_{\mathrm{U} 0126}=0.0001$. Effects of Chel, H89 and $\mathrm{U} 0126$ on the CP clock phase are depicted by open symbols. A full color version of this figure is available at https://doi.org/10.1530/JOE-20-0526.

expression of Gilz, which rose significantly already within $1 \mathrm{~h}(P=0.0173)$, then further increased being significantly elevated 2 and $4 \mathrm{~h}$ after the injection $(P<0.0001$ for both). The result confirmed the rapid responsiveness of the $\mathrm{CP}$ cells to DEX in vivo and thus served us as a proof of protocol validity for the in vivo experiments described in Figs 2 and $3 \mathrm{~A}$. We found that in the same CP samples, DEX injection resulted in an acute increase in Per1 expression which was first significant $2 \mathrm{~h}(P=0.0305)$ after the injection and remained elevated after $4 \mathrm{~h}(P=0.0221)$ (Fig. 3A). The expression of none of the other studied clock genes (Per2, Nr1d1, Bmal1 and E4bp4) showed any acute changes due to the DEX administration within the $4 \mathrm{~h}$ interval after the treatment (Fig. 3A). The results suggest that DEXinduced acute initiation of Per1 gene expression may be involved in the mechanisms of the CP clock resetting.

\section{DEX effect on the CP clock involves CRE-dependent pathways including PKA and MEK/ERK}

PKC and PKA kinases were previously found to participate in GC effects (reviewed in Adcock et al. 2002). Therefore, we tested the ability of specific inhibitors targeting components of these signaling cascades to block the DEX-induced phase shifts of the CP clock in vitro. We pretreated the organotypic CP explants with either one of the selected inhibitors of PKC and PKA cascades or the corresponding VEH before the DEX application (100 $\mathrm{nM}$ ) during the phase-delaying part of the PRC and compared the resulting shifts of the PER2bioluminescence rhythms (Fig. 3B). Pretreatment with PKC inhibitor chelerythrine (Chel; $10 \mu \mathrm{M}$ ) had no effect on the magnitude of the DEX-induced phase shifts 
A
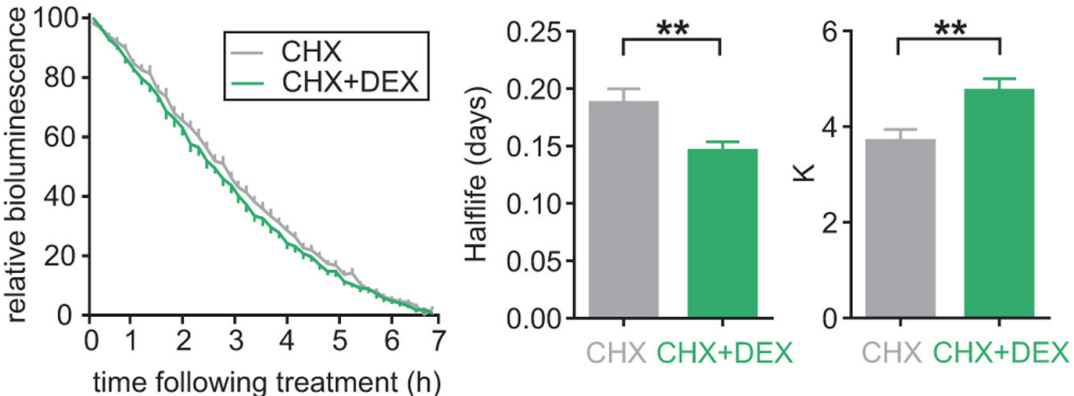

B
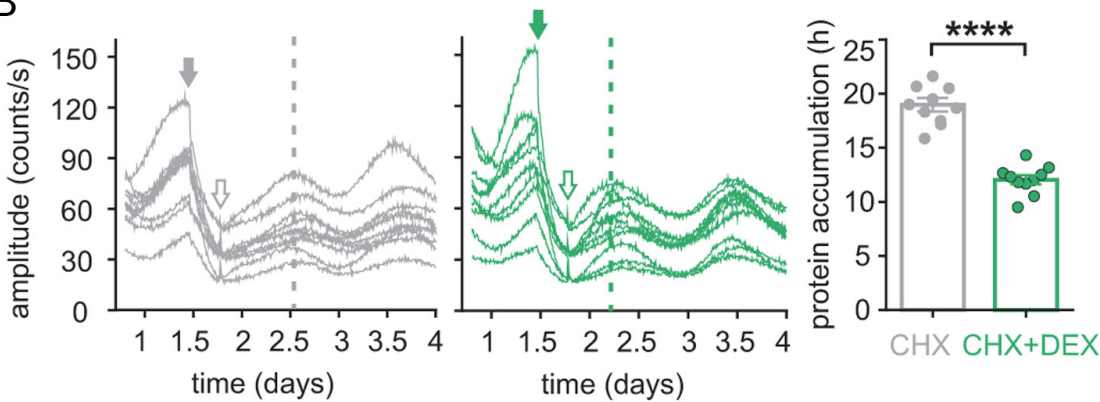

C
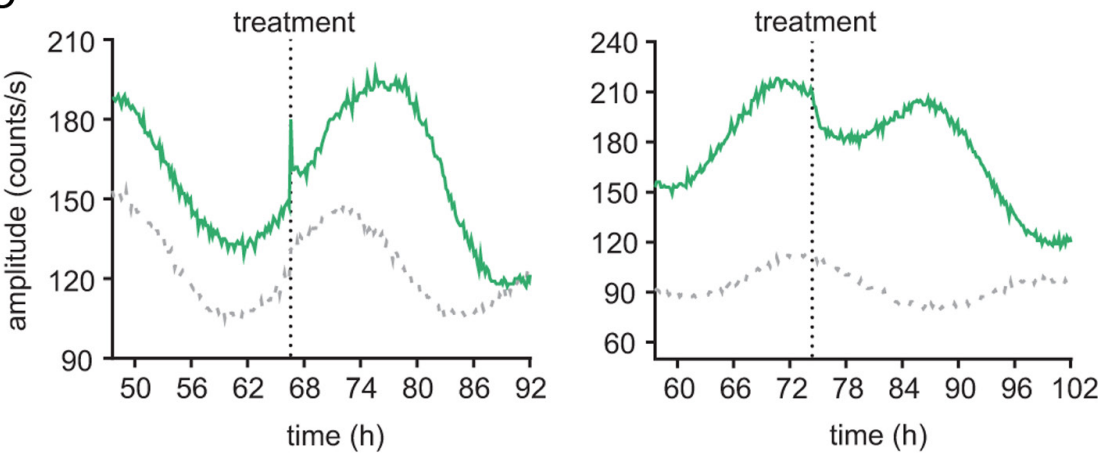

\section{Figure 4}

Effect of DEX on the turnover of PER2 protein in the $\mathrm{CP}$ explants. (A) The degradation of PER2 was measured using the decrease of bioluminescence following treatment with cycloheximide (CHX; $n=10)$ or with $\mathrm{CHX}+\mathrm{DEX}(n=10)$. One-phase exponential decay curves were fitted. $K$ and halflife were compared using t-test (mean \pm S.E.M.), ${ }^{*} P_{\text {halflife }}=0.0048, * \star P_{\mathrm{K}}=0.0026$. (B) Effect of DEX on PER2 protein accumulation in the CP explants. Bioluminescence traces of explants treated with $\mathrm{CHX}(n=10)$ or CHX+DEX $(n=10)$ are shown. The arrows indicate the time of treatment (full arrow) and wash (open arrow). The first bioluminescence peak after wash in CHX and CHX+DEX explants is shown (dashed lines). The comparison of PER2 protein accumulation rate (time necessary to reach the first peak) in $\mathrm{CHX/CHX+DEX}$ treated explants is shown (right graph; individual values and mean \pm s.E.M.). ${ }^{* \star \star *} P<0.0001$. (C) Acute responses of the bioluminescence traces to treatment. Representative bioluminescence traces of CP organotypic explants from mPer2Luc mice treated with either DEX or VEH after three days in culture. Vertical dotted lines mark the treatment time. Compared to VEH, DEX application during the PER2 protein rise (left graph) resulted in phase delay, whereas during the PER2 protein decline (right graph) caused a phase advance. Each trace corresponds to one organotypic explant. A full color version of this figure is available at https://doi.org/10.1530/ JOE-20-0526.
$(P=0.9581)$, whereas the PKA inhibitor H89 $(10 \mu \mathrm{M})$ slightly reduced these shifts $(P=0.0211)$. Importantly, inhibition of a downstream kinase, MEK, by U0126 (25 $\mu \mathrm{M})$ exhibited a highly significant reduction of the DEXinduced shifts $(P=0.0001)$. None of these inhibitors effective in blocking DEX-induced shifts induced phase shifts of the CP clock when applied on its own (Fig. 3C). The results demonstrate that the activation of the PKAERK1/2 pathway is partially involved in the effect of DEX on shifting the CP clock.

\section{DEX affects stability and accumulation rate of PER2 protein in CP}

To assess whether DEX affected the CP clock at the post-translational level, we examined its effects on PER2 protein accumulation and stability using CP organotypic explants of mPer2Luc mice. We treated the explants with translation inhibitor cycloheximide (CHX; $40 \mu \mathrm{g} / \mathrm{mL}$ ) to stop de novo translation and then analyzed the dynamics of PER2 degradation (decay rate of bioluminescence decline after CHX treatment) in the absence or presence of DEX (Fig. 4). The results revealed that DEX significantly shortened PER2 protein half-life $(P=0.0048)$ and increased $\mathrm{K}$ values $(P=0.0026)($ Fig. $4 \mathrm{~A})$. Additionally, after the termination of the $\mathrm{CHX}$-induced blockade of proteosynthesis, accumulation of de novo translated PER2 protein was significantly accelerated by DEX $(P<0.0001)$ (Fig. 4B). These results demonstrate that although DEX in the CP does not affect Per2 mRNA expression, it significantly modulates the dynamics of the PER2 protein degradation and accumulation, changing thus the protein turnover within the TTFL. Close examination of bioluminescence traces (Fig. 4C) revealed distinct acute responses of PER2 levels to DEX and VEH treatments. Compared to VEH, DEX application resulted 
in dramatic changes in the PER2-driven bioluminescence rhythm depending on its timing in relation to clock phase. Treatment with DEX during the rise of PER2 rhythm increased the rate and prolonged the interval of PER2 accumulation, resulting in a phase delay (Fig. 4C; left graph). Treatment with DEX performed after the PER2 peak accelerated the decrease of PER2 levels, resulting in phase advance of the rhythm (Fig. 4C; right graph).

\section{Discussion}

Our results provide compelling evidence that GCs play a crucial role in maintaining robustness and synchrony of the 4th ventricle CP clock with the actual activity/ rest cycle. In the in vivo study, $\mathrm{ADX}$ animals lacking endogenous GCs had significantly dampened clock gene rhythmicity in their $\mathrm{CP}$, and exposure of these animals to daily bouts of synthetic GC analog, DEX, reinforced the rhythmicity. The same effects we observed in vitro because DEX was able to increase the amplitude of the clock oscillation (strengthened the rhythm) and also efficiently shift the clock depending on the timing of its application. Finally, we found that DEX exhibited multiple effects on the CP clock, acutely inducing expression of Per1 mRNA and changing PER2 turnover via post-translational mechanisms, and that the PKA-ERK1/2 pathway is involved in modulation of some of the effects. Altogether, these effects are capable to mediate the role of GCs in strengthening and entraining the CP clock.

The presence of a circadian clock in the CP cells in vivo has been previously demonstrated (Quintela et al. 2015, 2018, Yamaguchi et al. 2020). In this study, we further extended the data by providing clock gene expression profiles of both positive and negative arms of the TTF in the CP with a higher temporal resolution than previously published (collecting samples in $4 \mathrm{~h}$ instead of 6-7 h intervals as in Quintela et al. 2015, Yamaguchi et al. 2020), allowing thus to determine their phases more accurately. We also confirmed the previously shown selfautonomous feature of the CP clock (Myung et al. 2018) via monitoring bioluminescence rhythms in organotypic explants prepared from $m P e r 2^{L u c}$ mice. In our set-up, the mean period of the CP clock was longer $(26.39 \pm 0.05 \mathrm{~h}$; mean \pm s.D.; $n=268$ ) compared to previously published data (Myung et al. 2018, Yamaguchi et al. 2020).

The role of the CP clock in the brain function has not been ascertained, although its control of rhythmic CSF production (Yamaguchi et al. 2020) and fine-tuning periods of other clocks in the brain, including the SCN (Myung et al. 2018, Yamaguchi et al. 2020), have been considered. For any of those functions, the CP clock needs to maintain the proper phase relative to the subject's activity state. We demonstrate that the CP employs rhythmic GC levels as the powerful signal to align its clock with activity/sleep cycles. The evidence is based on the demonstration that GCs affect the CP clock at multiple levels. We showed that all CP cells harbor GRs and, therefore, GCs may affect all CP cellular clocks simultaneously. The absence of endogenous GCs caused by the removal of adrenal glands dampened the clock gene expression rhythms in the $\mathrm{CP}$, and their supplementation by the synthetic analog DEX restored robustness of the rhythms. A similar approach has been previously used to ascertain the effect of GCs on the clock in neural cells of the extra-SCN brain regions (Amir et al. 2004, Lamont et al. 2005, Segall et al. 2006, Woodruff et al. 2016). However, the effect of GCs on the brain non-neural cells has not been considered. Importantly, we showed that both ADX alone, as well as ADX with DEX administration, affected the positive (Bmal1) and the negative (Per, Nr1d1) arms of the TTFL (Takahashi et al. 2008). Therefore, the robustness of the clock in $\mathrm{CP}$ seems to be conditional to the endogenous rhythm in GC levels. We found that DEX administration in vivo acutely induced Per1 gene expression in the CP, however, repeated DEX injections to ADX rats were not able to reinduce rhythmicity in its expression. This may be due to the slower metabolic turnover of DEX in comparison with that of native GC (Asare 2007), possibly resulting in DEX accumulation over time and continuous activation of Per1 GRE. DEX administration also shifted the phase of the clock gene expression profiles as compared to the SHAM and ADX groups. The profiles were phase delayed relative to the controls likely because we administered DEX every day at the time of activity onset, that is, when GCs already reached their maximal levels, but under physiological conditions, their levels started to rise well before waking up (Cheifetz 1971).

Importantly, we verified all the effects of DEX on the $\mathrm{CP}$ clock we have shown in vivo using in vitro monitoring of bioluminescence of CP organotypic explants of $m P e r 2^{\text {Luc }}$ mice. DEX not only increased amplitudes of these rhythms but also produced robust time-dependent phase-resetting of the clock. DEX treatments around the clock produced a typical type 0 PRC (Johnson 1992) with the phase shifts up to $12 \mathrm{~h}$ attained around the transition point matching the peak of the PER2-driven bioluminescence (time 12). The PRC shape is very similar to that for DEX in explants of various extra-brain clocks, for example, placenta 
(Čečmanová et al. 2019) and liver (Balsalobre et al. 2000). Notably, our results provide the first description of a PRC for a non-neuronal clock in the brain, demonstrating that the CP clock shares some properties with the nonneuronal clocks elsewhere in the body (Balsalobre et al. 2000). Importantly, the effect was specific to GCs because (1) the VEH treatment had only a minor effect, and (2) the GR antagonist mifepristone completely blocked the DEX-induced phase shifts. However, we must admit that the strong effect of $100 \mathrm{nM}$ DEX on the CP clock is not directly pertinent to physiological conditions where lower concentrations of endogenous corticosterone/cortisol fluctuating in pulses may have less robust effect on the $\mathrm{CP}$ clock than the sudden spike in the synthetic GR agonist. Nevertheless, the results still provide evidence that the CP clock is sensitive to the GR activation. Our data clearly show that the GCs may serve as a relevant signal resetting the CP clock in order to keep it in synchrony with the actual arousal state and suggest that therapeutic use of DEX may have unforeseen effects in the CNS.

The mechanism by which GCs entrain the circadian clocks in the body has been extensively studied and their interference with the TTFL components at various levels was demonstrated (Torra et al. 2000, Yamamoto et al. 2005, Nader et al. 2009, Lamia et al. 2011, Cheon et al. 2013, Murayama et al. 2019). Interestingly, these mechanisms seem to be tissue-dependent (Soták et al. 2016). The most common action of GCs is binding GRE in promoters of various genes inducing their rapid transcription (reviewed in Weikum et al. 2017). We found that the CP cells in vivo respond to DEX immediately by induction of GRE-responsive gene Glucocorticoid-induced leucine zipper (Gilz), which represents GC response shared by many tissues (Srinivasan \& Lahiri 2017). The GREs are present in promoters of several clock genes, namely Per1, Per2, and E4bp4 (Yamamoto et al. 2005, So et al. 2009, Cheon et al. 2013), however, in the CP only Per1 was acutely induced by DEX within the analyzed $4 \mathrm{~h}$ window. Notably, the acute response of Per1 expression to DEX treatment was lagging Gilz by at least $1 \mathrm{~h}$. One of the reasons for the apparent delay in the dynamics of induction of Per1 expression relative to Gilz might be the fact that the GRE-dependent activation of expression is modulated by additional mechanisms in the Per1 promoter, namely those related to activation of cAMP response element (CRE) (Tischkau et al. 2003). Such dual regulation is common in the circadian clock genes possessing both a CRE and GRE in their promoter (So et al. 2009, Cheon et al. 2013, Doi et al. 2019). Based on this, we cannot exclude the possibility that DEX increased also Per2 mRNA levels, but the induction was delayed to Per1 due to GRE/E-box sequence overlap and necessity for GR-BMAL1 interaction (Cheon et al. 2013). Involvement of CRE in the mechanism of DEX effects on the clock is plausible because previous studies have suggested the possibility of DEX binding on membrane-associated GRs, and subsequent modulation of PKA and PKC downstream signaling cascades (Groeneweg et al. 2012), and the PKAERK1/2 pathway is the most obvious target of activation of CRE on the gene promoters (Impey et al. 1998). Although GR-immunoreactivity is rather cytosolic in the CP, it still does not exclude a possibility of interplay between the GR and these signaling molecules. For example, blocking of PKA, MAPK, or PKC kinases suppressed GC-induced enhancement of thorn genesis in hippocampal CA3 region (Yoshiya et al. 2013). Additionally, previously described PKA-, MEK- or possibly ERK-mediated phosphorylation of cytosolic GR facilitating its translocation into nucleus, and/or phosphorylation of the GR co-factors forming the transcription complex to regulate GC-responsive genes, can be involved (reviewed in Adcock et al. 2002). Indeed, using inhibitors of the relevant signaling cascades, we found that phase shifts of the CP clock by DEX were partially dependent on functional PKA-ERK1/2 signaling. Although previous studies linked the effect of DEX with PKC activity (Groeneweg et al. 2012), the PKC inhibitor Chelerythrine had no effect. However, it does not completely exclude the participation of PKC pathway in the DEX effects on the CP clock because Chelerythrine selectivity as PKC inhibitor has been disputed (Davies et al. 2000). In contrast, H89 partially blocked the DEX responses, and the effect was even more pronounced after inhibition of the downstream MEK by U0126, suggesting the involvement of the PKA pathway. Importantly, none of the inhibitors effective in blocking the shifts (H89, U0126) induced significant phase shifts of the CP clock when administered on its own. Therefore, it is unlikely that the inhibitors affected the clock phase independent of DEX signaling. Nevertheless, it needs to be stressed that the conclusion on the involvement of the specific signaling pathways is weakened by limited selectivity of some of the inhibitors (Davies et al. 2000).

Additionally, using CHX-treated CP explants, we showed that DEX treatment changes PER2 protein turnover rate, shortening its half-life following the proteosynthesis inhibition by CHX and accelerating its de novo synthesis after CHX washout. These effects are consistent with the pattern of responses to DEX we observed in vitro that resulted in strong type 0 resetting of the CP clock. They represent a complex response manifested by a rapid 
change in PER2 levels followed by a change in the rate of PER2 synthesis that results in resetting of the CP clock (Lee et al. 2007).

Altogether, our results provide new insight into the complex and convergent molecular mechanisms of GC action on the clock in CP. They point at the importance of undisturbed GC rhythm for strengthening and entrainment of the clock in CP which is likely involved in modulation of internal brain environment across the day and night. Additionally, DEX is commonly used as a drug to treat a wide array of serious human diseases with reported side effects. Our results unraveled for the first time the extreme power of DEX treatment to reset the CP clock in dependence on the timing of the drug intake.

\section{Declaration of interest}

The authors declare that there is no conflict of interest that could be perceived as prejudicing the impartiality of the research reported.

\section{Funding}

The study was supported by the Czech Science Foundation grant P30412G069 (to A S), MEYS (LM2015062 Czech-Biolmaging), the project ERDF, OPPK BrainView CZ.2.16/3.1.00/21544, and the Research Project RV0: 67985823.

\section{Acknowledgements}

The authors thank Eva Suchanová for her technical assistance and to Prof Jiŕi Pácha and his colleagues for performing the surgery.

\section{References}

Adcock IM, Maneechotesuwan K \& Usmani O 2002 Molecular interactions between glucocorticoids and long-acting $\beta 2$-agonists. Journal of Allergy and Clinical Immunology 110 (Supplement) S261-S268. (https://doi.org/10.1067/mai.2002.129705)

Amir S, Lamont EW, Robinson B \& Stewart J 2004 A circadian rhythm in the expression of PERIOD2 protein reveals a novel SCNcontrolled oscillator in the oval nucleus of the bed nucleus of the stria terminalis. Journal of Neuroscience 24 781-790. (https://doi. org/10.1523/JNEUROSCI.4488-03.2004)

Asare K 2007 Diagnosis and treatment of adrenal insufficiency in the critically ill patient. Pharmacotherapy 27 1512-1528. (https://doi. org/10.1592/phco.27.11.1512)

Balsalobre A, Brown SA, Marcacci L, Tronche F, Kellendonk C, Reichardt HM, Schütz G \& Schibler U 2000 Resetting of circadian time in peripheral tissues by glucocorticoid signaling. Science $\mathbf{2 8 9}$ 2344-2347. (https://doi.org/10.1126/science.289.5488.2344)

Čečmanová V, Houdek P, Šuchmanová K, Sládek M \& Sumová A 2019 Development and entrainment of the fetal clock in the suprachiasmatic nuclei: the role of glucocorticoids. Journal of Biological Rhythms 34 307-322. (https://doi.org/10.1177/0748730419835360)
Cheifetz PN 1971 The daily rhythm of the secretion of corticotrophin and corticosterone in rats and mice. Journal of Endocrinology 49 xi-xii.

Cheon S, Park N, Cho S \& Kim K 2013 Glucocorticoid-mediated Period2 induction delays the phase of circadian rhythm. Nucleic Acids Research 41 6161-6174. (https://doi.org/10.1093/nar/gkt307)

Chiu C, Miller MC, Caralopoulos IN, Worden MS, Brinker T, Gordon ZN, Johanson CE \& Silverberg GD 2012 Temporal course of cerebrospinal fluid dynamics and amyloid accumulation in the aging rat brain from three to thirty months. Fluids and Barriers of the CNS 9 3. (https://doi. org/10.1186/2045-8118-9-3)

Davies SP, Reddy H, Caivano M \& Cohen P 2000 Specificity and mechanism of action of some commonly used protein kinase inhibitors. Biochemical Journal 351 95-105. (https://doi. org/10.1042/0264-6021:3510095).

Dibner C, Schibler U \& Albrecht U 2010 The mammalian circadian timing system: organization and coordination of central and peripheral clocks. Annual Review of Physiology 72 517-549. (https:// doi.org/10.1146/annurev-physiol-021909-135821)

Doi M, Shimatani H, Atobe Y, Murai I, Hayashi H, Takahashi Y, Fustin JM, Yamaguchi Y, Kiyonari H, Koike N, et al. 2019 Non-coding cis-element of Period2 is essential for maintaining organismal circadian behaviour and body temperature rhythmicity. Nature Communications 102563. (https://doi.org/10.1038/s41467-019-10532-2)

Groeneweg FL, Karst H, de Kloet ER \& Joëls M 2012 Mineralocorticoid and glucocorticoid receptors at the neuronal membrane, regulators of nongenomic corticosteroid signalling. Molecular and Cellular Endocrinology 350 299-309. (https://doi.org/10.1016/j. mce.2011.06.020)

Harrington MG, Salomon RM, Pogoda JM, Oborina E, Okey N, Johnson B, Schmidt D, Fonteh AN \& Dalleska NF 2010 Cerebrospinal fluid sodium rhythms. Cerebrospinal Fluid Research 7 3. (https://doi. org/10.1186/1743-8454-7-3)

Houdek P \& Sumová A 2014 In vivo initiation of clock gene expression rhythmicity in fetal rat suprachiasmatic nuclei. PLOS ONE 9 e107360. (https://doi.org/10.1371/journal.pone.0107360)

Impey S, Obrietan K, Wong ST, Poser S, Yano S, Wayman G, Deloulme JC, Chan G \& Storm DR 1998 Cross talk between ERK and PKA is required for $\mathrm{Ca} 2+$ stimulation of CREB-dependent transcription and ERK nuclear translocation. Neuron 21 869-883. (https://doi. org/10.1016/s0896-6273(00)80602-9)

Johnson CH 1992 Phase response curves: what can they tell us about circadian clocks. In Circadian Clocks from Cell to Human, pp. 209-249. Eds T Hiroshige \& K Honma. Sapporo: Hokkaido University Press.

Lamia KA, Papp SJ, Yu RT, Barish GD, Uhlenhaut NH, Jonker JW, Downes M \& Evans RM 2011 Cryptochromes mediate rhythmic repression of the glucocorticoid receptor. Nature $\mathbf{4 8 0} 552-556$. (https://doi.org/10.1038/nature10700)

Lamont EW, Robinson B, Stewart J \& Amir S 2005 The central and basolateral nuclei of the amygdala exhibit opposite diurnal rhythms of expression of the clock protein Period2. PNAS $1024180-4184$. (https://doi.org/10.1073/pnas.0500901102)

Lee B, Almad A, Butcher GQ \& Obrietan K 2007 Protein kinase C modulates the phase-delaying effects of light in the mammalian circadian clock. European Journal of Neuroscience 26 451-462. (https:// doi.org/10.1111/j.1460-9568.2007.05664.x)

Lindvall M \& Owman C 1981 Autonomic nerves in the mammalian choroid plexus and their influence on the formation of cerebrospinal fluid. Journal of Cerebral Blood Flow and Metabolism 1 245-266. (https://doi.org/10.1038/jcbfm.1981.30)

Martinho A, Gonçalves I, Costa M \& Santos CR 2012 Stress and glucocorticoids increase transthyretin expression in rat choroid plexus via mineralocorticoid and glucocorticoid receptors. Journal of Molecular Neuroscience 48 1-13. (https://doi.org/10.1007/s12031-012-9715-7)

Murayama Y, Yahagi N, Takeuchi Y, Aita Y, Mehrazad Saber Z, Wada N, Li EX, Piao X, Sawada Y, Shikama A, et al. 2019 Glucocorticoid https://joe.bioscientifica.com

https://doi.org/10.1530/JOE-20-0526 (c) 2021 Society for Endocrinology Published by Bioscientifica Ltd. Printed in Great Britain 
receptor suppresses gene expression of Rev-erb $\alpha(\mathrm{Nr} 1 \mathrm{~d} 1)$ through interaction with the CLOCK complex. FEBS Letters 593 423-432. (https://doi.org/10.1002/1873-3468.13328)

Myung J, Schmal C, Hong S, Tsukizawa Y, Rose P, Zhang Y, Holtzman MJ, De Schutter E, Herzel H, Bordyugov G, et al. 2018 The choroid plexus is an important circadian clock component. Nature Communications 9 1062. (https://doi.org/10.1038/s41467-018-03507-2)

Nader N, Chrousos GP \& Kino T 2009 Circadian rhythm transcription factor CLOCK regulates the transcriptional activity of the glucocorticoid receptor by acetylating its hinge region lysine cluster: potential physiological implications. FASEB Journal 23 1572-1583. (https://doi.org/10.1096/fj.08-117697)

Nilsson C, Stahlberg F, Thomsen C, Henriksen O, Herning M \& Owman C 1992 Circadian variation in human cerebrospinal fluid production measured by magnetic resonance imaging. American Journal of Physiology 262 R20-R24. (https://doi.org/10.1152/ajpregu.1992.262.1.R20)

Quintela T, Sousa C, Patriarca FM, Gonçalves I \& Santos CRA 2015 Gender associated circadian oscillations of the clock genes in rat choroid plexus. Brain Structure and Function 220 1251-1262. (https:// doi.org/10.1007/s00429-014-0720-1)

Quintela T, Albuquerque T, Lundkvist G, Carmine Belin A, Talhada D, Gonçalves I, Carro E \& Santos CRA 2018 The choroid plexus harbors a circadian oscillator modulated by estrogens. Chronobiology International 35 270-279. (https://doi.org/10.1080/07420528.2017.1400978)

Sathyanesan M, Girgenti MJ, Banasr M, Stone K, Bruce C, Guilchicek E, Wilczak-Havill K, Nairn A, Williams K, Sass S, et al. 2012 A molecular characterization of the choroid plexus and stress-induced gene regulation. Translational Psychiatry 2 e139-e139. (https://doi. org/10.1038/tp.2012.64)

Segall LA, Perrin JS, Walker CD, Stewart J \& Amir S 2006 Glucocorticoid rhythms control the rhythm of expression of the clock protein, Period2, in oval nucleus of the bed nucleus of the stria terminalis and central nucleus of the amygdala in rats. Neuroscience 140 753-757. (https://doi.org/10.1016/j.neuroscience.2006.03.037)

Silverberg GD, Mayo M, Saul T, Rubenstein E \& McGuire D 2003 Alzheimer's disease, normal-pressure hydrocephalus, and senescent changes in CSF circulatory physiology: a hypothesis. Lancet: Neurology 2 506-511. (https://doi.org/10.1016/s1474-4422(03)00487-3)

Sinclair AJ, Onyimba CU, Khosla P, Vijapurapu N, Tomlinson JW, Burdon MA, Stewart PM, Murray PI, Walker EA \& Rauz S 2007 Corticosteroids, 11ß-hydroxysteroid dehydrogenase isozymes and the rabbit choroid plexus. Journal of Neuroendocrinology 19 614-620. (https://doi.org/10.1111/j.1365-2826.2007.01569.x)

Skipor J \& Thiery JC 2008 The choroid plexus - cerebrospinal fluid system: undervaluated pathway of neuroendocrine signaling into the brain. Acta Neurobiologiae Experimentalis 68 414-428.

So AY-L, Bernal TU, Pillsbury ML, Yamamoto KR \& Feldman BJ 2009 Glucocorticoid regulation of the circadian clock modulates glucose homeostasis. PNAS 106 17582-17587. (https://doi.org/10.1073/ pnas.0909733106)

Soták M, Bryndová J, Ergang P, Vagnerová K, Kvapilová P, Vodička M, Pácha J \& Sumová A 2016 Peripheral circadian clocks are diversely affected by adrenalectomy. Chronobiology International 0528 1-10. (https://doi.org/10.3109/07420528.2016.1161643)

Spencer RL, Chun LE, Hartsock MJ \& Woodruff ER 2018 Glucocorticoid hormones are both a major circadian signal and major stress signal: how this shared signal contributes to a dynamic relationship between the circadian and stress systems. Frontiers in Neuroendocrinology 49 52-71. (https://doi.org/10.1016/j.yfrne.2017.12.005)

Srinivasan M \& Lahiri DK 2017 Glucocorticoid-induced leucine zipper in central nervous system health and disease. Molecular Neurobiology $\mathbf{5 4}$ 8063-8070. (https://doi.org/10.1007/s12035-016-0277-5)

Sumová A, Sládek M, Jáč M \& Illnerová H 2002 The circadian rhythm of Per1 gene product in the rat suprachiasmatic nucleus and its modulation by seasonal changes in daylength. Brain Research $\mathbf{9 4 7}$ 260-270. (https://doi.org/10.1016/s0006-8993(02)02933-5)

Sumová A, Jác M, Sládek M, Šauman I \& Illnerová H 2003 Clock gene daily profiles and their phase relationship in the rat suprachiasmatic nucleus are affected by photoperiod. Journal of Biological Rhythms $\mathbf{1 8}$ 134-144. (https://doi.org/10.1177/0748730403251801)

Takahashi JS, Hong HK, Ko CH \& McDearmon EL 2008 The genetics of mammalian circadian order and disorder: implications for physiology and disease. Nature Reviews: Genetics 9 764-775. (https://doi. org/10.1038/nrg2430)

Tischkau SA, Mitchell JW, Tyan SH, Buchanan GF \& Gillette MU 2003 $\mathrm{Ca} 2+/$ cAMP response element-binding protein (CREB)-dependent activation of Per1 is required for light-induced signaling in the suprachiasmatic nucleus circadian clock. Journal of Biological Chemistry 278 718-723. (https://doi.org/10.1074/jbc.M209241200)

Torra IP, Tsibulsky V, Delaunay F, Saladin R, Laudet V, Fruchart JC, Kosykh V \& Staels B 2000 Circadian and glucocorticoid regulation of Rev-erbo expression in liver. Endocrinology 141 3799-3806. (https:// doi.org/10.1210/endo.141.10.7708)

Weikum ER, Knuesel MT, Ortlund EA \& Yamamoto KR 2017 Glucocorticoid receptor control of transcription: precision and plasticity via allostery. Nature Reviews: Molecular Cell Biology 18 159-174. (https://doi.org/10.1038/nrm.2016.152)

Wolburg H \& Paulus W 2010 Choroid plexus: biology and pathology. Acta Neuropathologica 119 75-88. (https://doi.org/10.1007/s00401009-0627-8)

Woodruff ER, Chun LE, Hinds LR \& Spencer RL 2016 Diurnal corticosterone presence and phase modulate clock gene expression in the male rat prefrontal cortex. Endocrinology 157 1522-1534. (https:// doi.org/10.1210/en.2015-1884)

Wostyn P, Audenaert K \& De Deyn PP 2011 Choroidal proteins involved in cerebrospinal fluid production may be potential drug targets for Alzheimer's disease therapy. Perspectives in Medicinal Chemistry 5 11-17. (https://doi.org/10.4137/PMC.S6509)

Yamaguchi T, Hamada T, Matsuzaki T \& Iijima N 2020 Characterization of the circadian oscillator in the choroid plexus of rats. Biochemical and Biophysical Research Communications 524 497-501. (https://doi. org/10.1016/j.bbrc.2020.01.125)

Yamamoto T, Nakahata Y, Tanaka M, Yoshida M, Soma H, Shinohara K, Yasuda A, Mamine T \& Takumi T 2005 Acute physical stress elevates mouse Period1 mRNA expression in mouse peripheral tissues via a glucocorticoid-responsive element. Journal of Biological Chemistry 280 42036-42043. (https://doi.org/10.1074/jbc.M509600200)

Yoshiya M, Komatsuzaki Y, Hojo Y, Ikeda M, Mukai H, Hatanaka Y, Murakami G, Kawata M, Kimoto T \& Kawato S 2013 Corticosterone rapidly increases thorns of CA3 neurons via synaptic/extranuclear glucocorticoid receptor in rat hippocampus. Frontiers in Neural Circuits 7 191. (https://doi.org/10.3389/fncir.2013.00191)

Received in final form 1 December 2020

Accepted 18 December 2020

Accepted Manuscript published online 23 December 2020 https://joe.bioscientifica.com https://doi.org/10.1530/JOE-20-0526
(C) 2021 Society for Endocrinology Published by Bioscientifica Ltd.
Printed in Great Britain 